\title{
Ya. B. Zeldovich (1914-1987) Chemist, Nuclear Physicist, Cosmologist
}

\author{
Varun Sahni \\ Inter-University Centre for Astronomy and Astrophysics, \\ Post Bag 4, Ganeshkhind, Pune 411 007, India \\ email: varun@iucaa.ernet.in
}

\section{Introduction}

Yakov Borisovich Zeldovich was remarkably talented. $\dagger$ His active scientific career included major contributions in fields as diverse as chemical physics (adsorption \& catalysis), the theory of shock waves, thermal explosions, the theory of flame propogation, the theory of combustion \& detonation, nuclear \& particle physics, and, during the latter part of his life: gravitation, astrophysics and cosmology (Zeldovich 1992).

Zeldovich made key contributions in all these area's, nurturing a creative and thriving scientific community in the process. His total scientific output exceeds 500 research article and 20 books. Indeed, after meeting him, the famous English physicist Stephen Hawking wrote "Now I know that you are a real person and not a group of scientists like the Bourbaki".

Remarkably Zeldovich never received any formal university education! He graduated from high school in St. Petersburg at the age of 15 after which he joined the Institute for Mechanical Processing of Useful Minerals ('Mekhanabor') to train as a laboratory assistant. The depth of Zeldovich 's questioning and his deep interest in science soon reached senior members of the scientific community and, in 1931, the influential soviet scientist A.F. Ioffe wrote a letter to Mekhanabor requesting that Zeldovich be "released to science". Zeldovich defended his $\mathrm{PhD}$ in 1936 and, years later, reminiscenced of the "happy times when permission to defend [a $\mathrm{PhD}$ ] was granted to people who had no higher education".

In the 1930's, having done pathbreaking work on combustion and detonation (there is a 'Zeldovich number' in combustion theory), Zeldovich moved to nuclear physics writing seminal papers demonstrating the possibility of controlled fission chain reactions among uranium isotopes. This was the time when fascism was on the rise in Germany, and, in an effort for national survival, the Soviet Union was developing its own atomic program of which Zeldovich (then in his mid 20's) quickly became a key member. According to Andrei Sakharov, "from the very beginning of Soviet work on the atomic (and later thermonuclear) problem, Zeldovich was at the very epicenter of events. His role there was completely exceptional" (Sakharov 1988). One might add that Zeldovich 's earlier work on combustion paved the way for creating the internal ballistics of solid-fuel rockets which formed the basis of the Soviet missile program during the 'great patriotic war' and after Ginzburg (1994). After the war Zeldovich went on to do pioneering work in several other aspects of nuclear and particle physics.

$\dagger$ This brief presentation is based on arXiv:1403.1537. 


\section{Astrophysics}

Zeldovich decided to change course midstream and, from about 1964, devoted his phenomenal abilities to problems in astrophysics. Below I (imperfectly) summarize some of Zeldovich's seminal contributions to astrophysics and cosmology.

- In 1962 Zeldovich showed that a black hole could be formed not only during the course of a stellar explosion but by any mechanism which compressed matter to sufficiently high densities. This opened up the possibility of the formation of microscopically small black holes in the early Universe. In 1964 Zeldovich suggested that a black hole may be detected by its influence on the surrounding gas which would accrete onto the hole. Zeldovich also suggested that one could look for a black hole in binary star systems through the holes influence on the motion of its bright stellar companion.

Zeldovich (1971) and his student Starobinsky (1973) showed that under certain conditions a rotating black hole could loose energy via the production of a particle-antiparticle pair. These papers were precursors of later work including Stephen Hawking's famous paper on evaporating black holes published in 1975. Zeldovich and Starobinsky also did a seminal study of particle production in the early universe.

- In 1966 Zeldovich and Gershtein showed that massive neutrino's could play a key cosmological role thus paving the way for the concept of non-baryonic dark matter. Zeldovich appreciated the enormous impact that stable topological defects could have within a cosmological setting. He and his colleagues demonstrated that whereas monopoles and domain walls were disastrous for cosmology, cosmic strings might be useful since they would act as 'seeds' onto which matter accreted resulting in the formation of gravitationally bound systems.

- In 1967 Zeldovich applied himself to the issue of the cosmological constant ' $\Lambda$ '. Originally introduced by Einstein in 1917, the cosmological constant has the unusual property that, unlike other forms of matter, its pressure is negative and equal, in absolute terms, to its density $(P=-\rho)$. Hence, while the density in normal forms of matter declines in an expanding universe, the density in $\Lambda$ remains frozen to a constant value $\rho=\Lambda / 8 \pi G$. After its inception the cosmological constant had fallen into disrepute since it did not seem to be required by observations. Even Einstein distanced himself from it, calling the $\Lambda$-term 'my biggest blunder'. Zeldovich radically changed this perspective by persuasively arguing that, within the context of quantum field theory, the prospect of a non-zero value for the $\Lambda$-term should be taken extremely seriously. The reason is that the quantum polarization of the vacuum results in a vacuum energy which, quite remarkably, has the precise form of a cosmological constant: $\left\langle T_{i}^{k}\right\rangle=\Lambda \delta_{i}^{k} / 8 \pi G$. By providing a physical basis for the $\Lambda$-term, Zeldovich paved the way for future advances including cosmic inflation and dark energy.

- Zeldovich himself felt that his main contribution to cosmology was in the understanding of gravitational instability. In 1972 Zeldovich showed how primordial fluctuations could have a scale invariant spectrum. A decade later, the scale invariant spectrum was shown to be a generic prediction of Inflationary models of the early Universe.

In 1970 Zeldovich proposed a remarkably simple approximation which could be used to follow a perturbation from its initially linear form into the fully nonlinear regime when it gave rise to the formation of 'pancakes'. Along the way Zeldovich upset a widely prevailing world view according to which the assembly of the first large astrophysical objects in the universe was spherical in nature.

- In 1972, Zeldovich and Sunyaev published a seminal paper in which they showed that photons from the cosmic microwave background would scatter off the hot plasma 


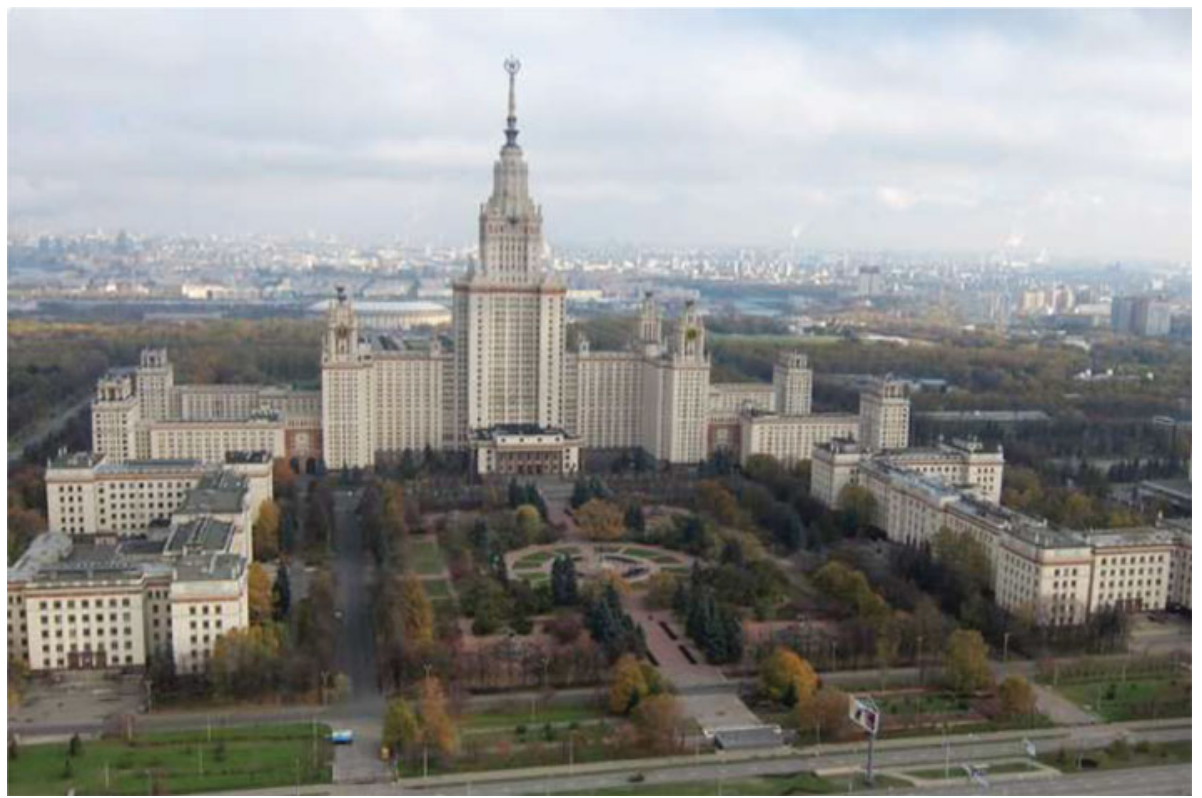

Figure 1. Moscow State University. The central building and its wings contained the hostel as well as the department of maths, the main library etc. The right building in the forefront housed the physics department where Zeldovich taught.

trapped in the deep potential wells of clusters. This would alter the brightness of the CMB when viewed in the direction of a cluster.

\section{Personal Reminiscences}

My first meeting with Zeldovich took place in 1978 when, as a student of physics at Moscow State University (see fig. 2), I was looking for a professor who would guide me for my (pre-MSc) course work. Zeldovich agreed and, to test my skills, gave me a project in general relativity, a subject which I had just started to learn. I soon realized that Zeldovich was an excellent teacher and could explain in very simple language exceedingly complex physical ideas. This was a great boon to students since, despite his extremely busy scientific schedule, Zeldovich always found time to teach courses at Moscow University. So it was that I was initiated into the intricate and beautiful field of cosmology via a twenty two lecture course taught by Zeldovich - each lecture being of roughly 2 hour duration with a ten minute break in between.

Zeldovich continuously modified and expanded his course material taking care to ensure that significantly new developments in the field were covered. (On attending the very same cosmology course 4 years later, I was pleasantly surprised at finding that almost a quarter of its content was new. Zeldovich assiduously incorporated several recent developments including grand unification, topological defects and inflation, into his 'selfrevised' syllabus.) Participants at his lectures consisted not only of students, but also senior researchers and professors, many of whom stayed back after class to discuss new science ideas with Zeldovich. Another remarkable quality of Zeldovich was his willingness to learn from others and to acknowledge, often in public, the mistakes which he had made, and what could be learned from them. Thus he admitted in class how he had misunderstood the data regarding the cosmic microwave background in the early 1960's 
and, to his great regret, had initially advocated the cold big bang model instead of the hot one.

Zeldovich strove to explain complicated ideas simply through numerous entertaining articles and text books (Zeldovich (1992); Zeldovich \& Novikov (1971, 1996)) including his excellent monograph 'Higher Mathematics for Beginners' Zeldovich (1970) and Zeldovich (1976) which presented serious mathematical ideas in a form which is accessible to a high school student. He once wrote "the so-called 'strict' proofs and definitions are far more complicated than the intuitive approach to derivatives and integrals. As a result, the mathematical ideas necessary for an understanding of physics reach school-pupils too late. It is like serving the salt and pepper not for lunch, but later - for afternoon tea".

Indeed, the key role played by the science popularisation program for Soviet science cannot be over-emphasised. Talented scientists and mathematicians took great pains in bringing the joy of their disciplines to a much younger audience, including high school students. I believe the great heights reached by the Soviet science school owed much to this popularisation effort, which created an enormous base from which talent could be drawn for the construction of the impressive Soviet science pyramid at the very apex of which belonged Landau, Zeldovich, Kolmogorov and other great thinkers. It might be appropriate to draw here an analogy with the Soviet chess school which also had an enormous grass-root level base, from which rose a formidable range of grandmasters and world champions.

Zeldovich's manner of conducting exams was also quite unusual ! When, towards the end of his cosmology course the time came for exams, I realised that there was no formal time table for a written test, as was usually the case. Instead Zeldovich asked me to meet him outside his office a few days later. When I did, Zeldovich scribbled two problems and asked me to solve them, after which he walked away. Although I had gone through his course material diligently I could not, even after trying hard, figure out how either of these problems could be solved, and so feeling quite dejected I walked back to my hostel a few miles away. This was summer 1979. By winter, with the heavy snows of Moscow having set in, I managed to crack one of the problems and, feeling rather elated, went back to Zeldovich. Zeldovich subjected me to a strenuous viva-voce after which he declared that I had passed with an A and, even more significantly, that my solution to his problem was new and original and could be published as a paper.

I should add that while most people found Zeldovich to be very inspiring, there were some who found his intellectual brilliance rather intimidating. Indeed Zeldovich did not suffer fools lightly and I have seen him demolish in less than a few minutes many a senior scientist propounding a silly idea. On the other hand, Sakharov mentions that Zeldovich's " effect on his pupils was remarkable; he often discovered in them a capacity for scientific creativity which without him would not have been realized or could have been realized only in part and with great difficulty." In this he was following the tradition of the other great Russian guru, Landau, who like Zeldovich, fostered and left behind a great scientific legacy in the form of a school of physics which was almost unique in scientific method and style.

Around this time I made friends with Lev Kofman. Lev was originally from Tallinn but went to school in Tartu. He had been denied entry into a leading Moscow institution on the pretext that his eyesight was weak. Probably this was not the real reason, but despite this setback Lev was adamant to learn cosmology from the great theorists in Moscow. Lev and I soon became close friends and whenever Lev visited Moscow he stayed with me in the Moscow University hostel. Since Lev did not have a moskovskaya propiska (permission to legally live in Moscow) his stay at the hostel was an act of subterfuge and had to be carefully hidden from the authorities. This meant that he could not enter 


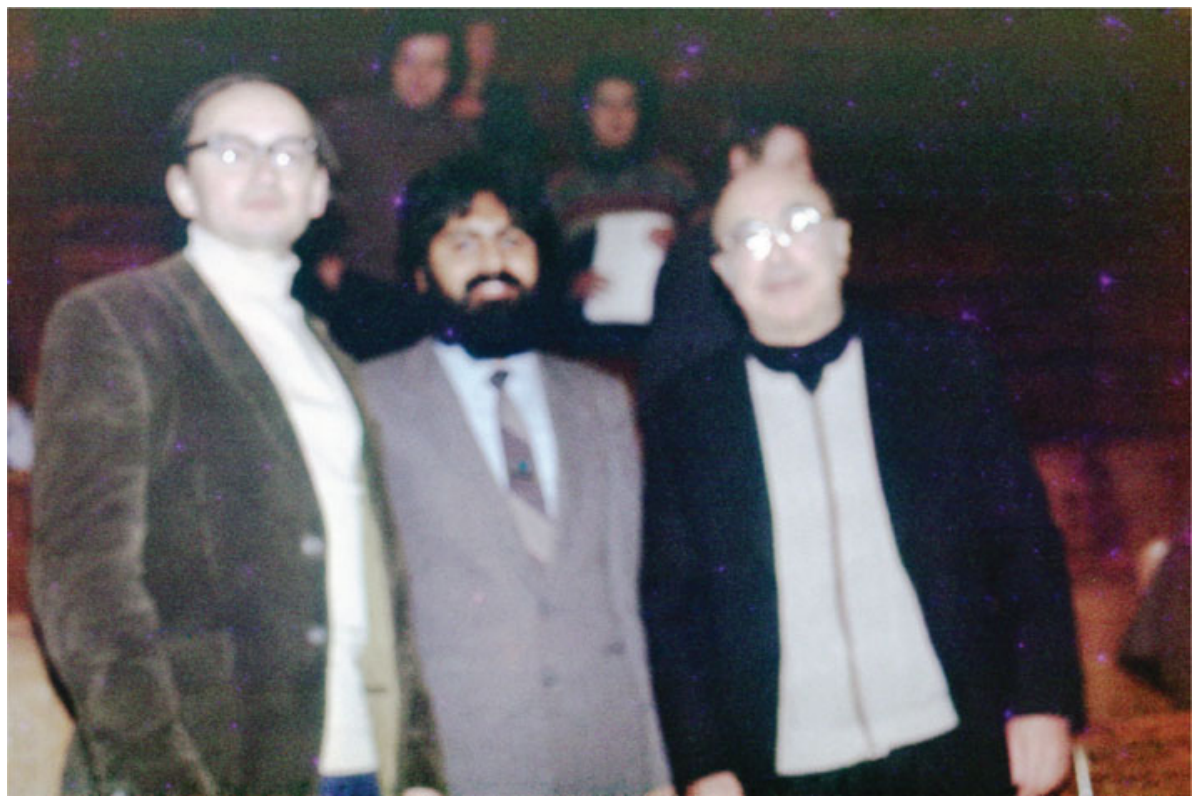

Figure 2. With my two gurus Starobinsky (left) and Zeldovich (right) at my $\mathrm{PhD}$ defence in Moscow (1985). One of the few times in my life that I wore a suit (which was borrowed).

the university like a normal person but had to climb a four meter high iron fence which surrounded our hostel, a hazardous task especially during winter, when both ground and fence were coated with ice.

Occasionally Lev did get caught. On one such occasion an inspection committee raided my room early one morning and took Lev away to the police precinct. I was very alarmed since I did not want anything nasty to happen to my good friend, so I ran after the police. I rescued Lev by telling the police officer, who happened to be a decent fellow, that we were both students of Academician Zeldovich whose classes would begin that morning, and that it would be a shame if Lev, who had travelled all the way from Estonia for this, should miss them. I guess my persuasive skills were at their best, since the policeman let Lev go, and we immediately ran to the physics building to listen to Zeldovichs lecture on cosmology (without any breakfast). Figure 2 gives an impression of the building complex of Moscow State University.

I might mention here that while Zeldovich was pretty tough when it came to science, on the personal front he was very generous. I experienced Zeldovich empathy on many occasions. Some years after commencing work on my PhD I was shocked to hear that that Zeldovich 's wife had just died of a serious illness. Together with some friends I went to pay my respects and offer condolences. Even before I could utter a word, Zeldovich turned to me and offered his own profuse condolences on the assasination of Indira Gandhi who had lost her life to extremists the very same day that Zeldovich 's wife lost hers to illness. In his eyes I could see how deeply he felt, and I was profoundly moved that he could place the historical anguish of a neighboring nation on the same footing as his own very deep and personal loss.

Although very fond of travel, Zeldovich faced numerous travel restrictions due to his early involvement with the Soviet defence program. In 1982, perhaps because of my political innocence and youthful enthusiasm, I was very keen that Zeldovich visit India, and before embarking home for holidays asked him what I could do to ensure his visit. 


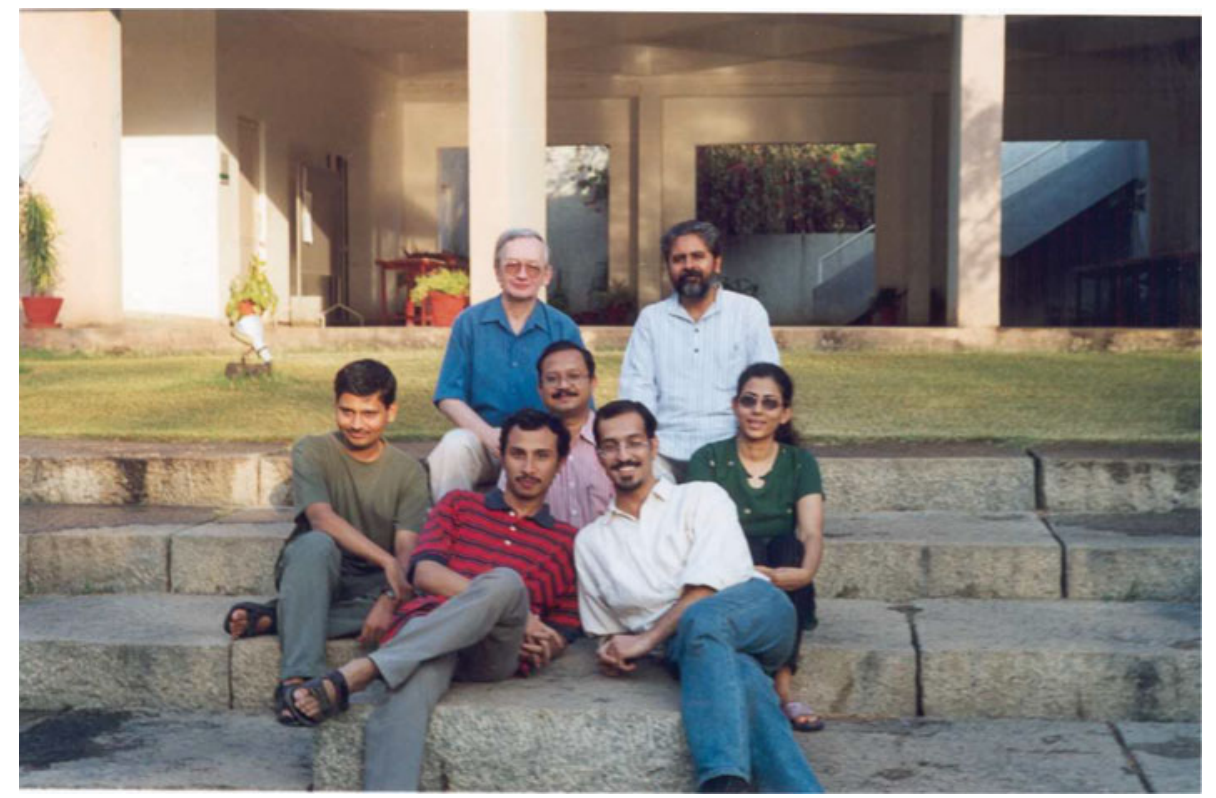

Figure 3. Some members of the Zeldovich school at IUCAA. Alexei Starobinsky and Varun Sahni (top row), Tarun Souradeep (middle), Jatush Sheth, Sanjit Mitra, Amir Hajian and Ujjaini Alam (bottom row).

Looking at me wistfully Zeldovich remarked, "they (the soviet authoroties) will reply to your governments invitation saying Zeldovich is ill and unable to travel, but look at me (Zeldovich flexed his muscles) I am perfectly fit and can travel tomorrow !". Indeed Zeldovich 's visit to India never did materialise and the following year I bore witness to the very bizarre policies of the Soviet government with regard to foreign travel by its eminent scientists. Zeldovich and Starobinsky had both been invited to travel to a famous meeting on general relativity (GR10) scheduled in Padova, Italy in 1983. Indeed, Zeldovich had been invited to organise a special session on the Early Universe while Starobinsky had consented to give a plenary talk on Inflation, which was then a very hot topic. I too was planning to go since my paper (written jointly with Starobinsky and another of his pupils Lev Kofman) had been accepted for presentation. I was very excited by the prospect of travelling to Italy from Moscow (3 days journey by train passing through several countries) and the day before leaving I met Starobinsky who took me to Zeldovich with the remark "Yakov Borisovich here is Varun, the only member of our delegation who is sure of travelling to Italy" ! I was not a little surprised to learn that, with only four days remaining for the meeting, neither Zeldovich nor Starobinsky had as yet received official sanction for their visit. I was broken hearted when, on reaching Padova 4 days later, I learned that neither of my guru's had been allowed to travel, and that the organisers were in a quandary as to how to salvage the early universe session. What was even more disheartening was the fact that while neither Zeldovich nor Starobinsky had been allowed to make the journey to Italy the official Soviet delegation was replete with scientistsc of rather mediocre quality but with deep party connections, who despite their ideological leanings were not ashamed to thoroughly enjoy 'capitalist hospitality'. 
Viewed in retrospect, and with the demise of the Soviet Union, it appears quite incomprehensible as to why many of its leading scientists could not travel abroad and enjoy an envigorating science discussion with their contemporaries in the east and west.

\section{Back in India}

After obtaining my $\mathrm{PhD}$ (in 1985 - see fig. 2) I returned to India (via post-docs in the UK and Canada) where I helped in the setting up of a new institution IUCAA. At the time of my return (1991) IUCAA consisted of little more than a tin-roofed shed surrounded by an enormous number of trees, generously populated by birds, snakes and on the ground, even scorpions. We set about building this new institution (under the leadership of Prof. Jayant Narlikar) with great enthusiasm, and in just a few years Sergei Shandarin and I organized the first Zeldovich meeting on large scale structure.

The Zeldovich legacy endures at IUCAA, where Tarun Souradeep (my first PhD student and now an IUCAA faculty) and I have, between us, successfully guided over a dozen cosmology PhDs, several of whom have also been mentored by Alexei Starobinsky, a frequent visitor to India (see fig. 3).

\section{References}

Ginsburg, V. L. 1994, Yakov Borissovich Zel'dovich, Biographical Memoirs of Fellows of the Royal Society 40, 431-441

Sakharov, A. 1988, A man of universal interests, Nature, 331, 671

Zeldovich, Ya. B. 1970, Higher mathematics for beginners, Nauka, Moscow

Zeldovich, Ya.B.\& Myskis, A. D. 1976 Elements of Applied Mathematics, Mir Publishers, Moscow

Zeldovich, Ya.B. 1992, Selected works of Yakov Borisovich Zeldovich, Vol. 1 \& 2, Princeton University Press

Zeldovich, Ya. B. 1992, My Universe, selected reviews, Harwood academic

Zeldovich, Ya.B. \& Novikov, I. D. 1971, Relativistic Astrophysics, Vol. 2: The Structure and Evolution of the Universe, Chicago, IL: University of Chicago Press

Zeldovich, Ya.B. \& Novikov, I. D. 1996, Relativistic Astrophysics, Vol. 1: Stars and Relativity, Mineola, NY: Dover Publications 